\title{
Optimum Polarization for Foliage Camouflaged Target Discrimination Using a Genetic Algorithm
}

\author{
Mojtaba Dehmollaian* and Kamal Sarabandi \\ Radiation Laboratory \\ Department of Electrical Engineering and Computer Science \\ The University of Michigan, Ann Arbor, MI 48109-2122 \\ E-mail: saraband@eecs.umich.edu
}

\begin{abstract}
The coherent scattering model can be run for many realizations of foliage around a hard target to obtain the statistical variations of foliage and target response in a Monte Carlo simulation. Foliage camouflaged target detection at low microwave frequencies is investigated, using a powerful foliage and hard target simulation tool which accounts for near-field interactions between the hard target and foliage. Based on fully polarimetric simulation results of a foliage camouflaged metallic target a polarization synthesis optimization method for improving signal to clutter is examined. A genetic algorithm is implemented for finding an optimal set of radar transmit and receive polarizations that maximizes target backscatter to clutter ratio.
\end{abstract}

\section{INTRODUCTION}

A very comprehensive physics-based foliage and target scattering model has recently been developed that can account for near field interactions among foliage, ground, and the target [1],[2]. In this model, individual tree in forest stand is decomposed into its basic constituents, trunk, branches and leaves which are respectively modeled by dielectric cylinders and thin dielectric disks, whose dielectric constants are functions of moisture content, frequency, and temperature [5]. Structure of trees are preserved using a statistical Lindenmayer system which can generate very complex tree structures with a finite number of structural and botanical parameters. Next, single scattering theory, which also includes the interaction of the scatterers with the ground plane, is applied to compute the scattering from and propagation through the forest canopy. The ground plane is modeled by a dielectric half-space whose dielectric constant is determined based on the frequency, soil moisture content, and percentage of sand and clay. The field at a given point inside the foliage is obtained by coherent addition of all the scattering contributions from all adjacent tree components, illuminated by the mean field, and its reflection from the ground. This is in addition to the direct field, attenuated by the foliage (mean field) and its reflection from the ground that directly goes through the foliage. The mean field is computed using Foldy's approximation, which accounts for the phase change as well as extinction due to the scattering and absorption of the tree particles. Scattering from large scatterers are formulated in a uniform fashion that allows field computation from the scatterer near-field to far-field region [2]. Using this foliage model, a hybrid target-foliage model is then developed for 
investigating the scattering behavior of metallic hard targets embedded inside a forest canopy, at high frequencies [4]. Reciprocity theorem is used very effectively to compute the backscattered field using the incident fields on the target, and induced electric currents. In this hybrid model, calculation of induced electric currents on the target is done using an efficient iterative Physical Optics (PO) and Geometrical Optics (GO) approximations. The problem of particular interest is to discriminate the target backscattering response from that of the clutter. One way of achieving this, is to investigate the polarization responses of the foliage, and the foliage embedded target and examine whether there exists a particular set of transmit and receive polarizations that maximizes the signal to clutter in the statistical sense. An optimization procedure is needed to find the optimal radar polarization configuration for different clutter realizations and keeping the same target inside the clutter. Because of the discontinuous nature and the large number of local minima of the cost function a genetic algorithm (GA) is chosen for the optimization procedure. In past GA has been successfully used to characterize the optimum polarization for multiple target discrimination [3]. The advantage of GA is that it is not a gradientbased approach and the desired objective function is globally optimized. In this paper, we first summarize the backscattering computation formulation of metallic hard targets inside the foliage. Next the implementation of a GA optimization procedure for finding the optimum polarization that suppresses the effect of foliage in backscattering response of a target inside the forest, is addressed.

\section{THEORY}

Using reciprocity theorem it can be shown that each component of the backscattered field can be computed from [4]

$$
\mathbf{S}_{p q}= \pm \frac{i k_{o} Z_{o}}{4 \pi} \int_{\mathbf{S}} \bar{E}_{1 p} \cdot \bar{J}_{2 q} d s^{\prime}
$$

where subscript $p$ and $q$ can be $h$ or $v$ for horizontal and vertical polarizations. The first and second subscripts denote the receive and the transmit polarizations, respectively. In (1), $\bar{E}_{1 p}$ is the $p$-component of the total incident field on the target, and $\bar{J}_{2 q}$ is the electric current on the surface of the hard target, induced by the $q-$ polarized incident wave. For electrically large objects, PO approximation can provide very accurate scattered field near specular directions. In a forest with many scatterers around, many specular points can be obtained. In this approach the challenging problem is how to determine the shadow and lit regions on a complex target. This problem drastically exasperated for a target inside the forest being illuminated by a very large number of scatterers around. Thus, it is almost impossible to apply a ray tracing method. Noting that the shadowed regions are produced as a result of scattering in forward direction, we can implement an efficient iterative approach using the magnetic field integral (MFIE) equation, used to iterate on first order PO. The induced electric current $\bar{J}(\bar{r})$ on a metallic object, can be obtained from

$$
\bar{J}(\bar{r})=2 \hat{n} \times \bar{H}_{i}(\bar{r})+2 \hat{n} \times \int_{\mathbf{S}}\left(\bar{r}-\bar{r}^{\prime}\right) \times \bar{J}\left(r^{\prime}\right)\left(i k_{0}-\frac{1}{\left|\bar{r}-\bar{r}^{\prime}\right|}\right) \frac{e^{i k_{0}\left|\bar{r}-\bar{r}^{\prime}\right|}}{4 \pi\left|\bar{r}-\bar{r}^{\prime}\right|^{2}} d \bar{r}^{\prime}
$$

It is noted that, if the surface of the object is smooth enough, the integral equation given by (2) can be solved iteratively. If the radii of curvature is small the integral part is small, therefore, to the first order approximation $\bar{J}^{(1)}=2 \hat{n} \times \bar{H}_{i}$, (the 


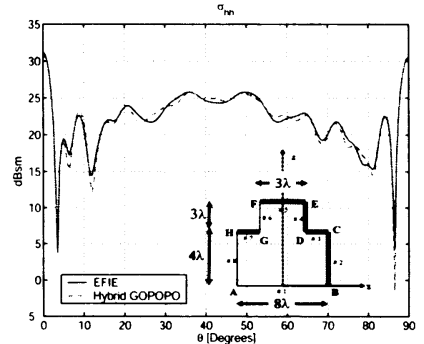

(a)

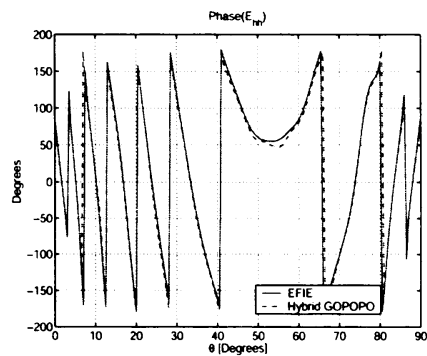

(b)

Fig. 1. Comparison of backscattering RCS calculated by exact EFIE method and Hybrid GOPO for a metallic target shown, at frequency of $2 \mathrm{GHz}, \phi=180^{\circ}$, and different elevation angles $\theta$. (a) $\sigma_{h h}$ (b) Phase $\left(E_{h h}\right)$.

PO current is dominant). Higher order currents can be obtained using, $\bar{J}^{(m)}=$ $2 \hat{n} \times L\left[\bar{J}^{(m-1)}\right]$, where $L\left[\bar{J}^{(m-1)}\right]$ is the surface integral evaluated for $(m-1)^{t h}$ order current $\bar{J}^{(m-1)}$. To calculate the field on the target for every source point, a simple geometrical optics (GO) shadowing condition given by $\hat{k} \cdot \hat{n}<0$ is used, where $\hat{k}$ is a unit vector from the source to the point on the surface, and $\hat{n}$ is a unit normal on the target. The same approach is implemented through the iteration. That is, in computation of (2), only the contribution of the points where,

$$
\left(\bar{r}-\bar{r}^{\prime}\right) \cdot \hat{n}<0
$$

is considered. This method, referred to as Hybrid GOPOPO, is applied to a target, shown in Fig. 1. The length of the target is $10 \lambda$ along $\hat{y}$ direction, and a radar operating frequency of $2 \mathrm{GHz}$ is assumed. Also the scattering problem is solved using MoM. A very good agreement is shown between exact electric field integral equation (EFIE) calculation, and the hybrid method. The advantage of this method becomes more clear when we put the object inside the forest canopy. In that case, for every scatterer and every point on the target only a simple condition $(\hat{k} \cdot \hat{n}<0)$ is checked where as for ray tracing approach every ray should be tracked. The iteration process on the other hand converges fast, and takes the effect of shadowing and multiple scattering on the target into account simultaneously.

\section{Polarization Optimization}

In order to suppress the backscattering response of clutter, a polarization optimization procedure is considered. To maximize the ratio of the backscatter RCS from the target to that of the clutter a Monte Carlo simulation for a fully polarimetric radar observing the target in a pine forest is carried out. In this Monte Carlo simulation an optimum polarization configuration which gives the maximum signal-to-clutter ratio, for all realizations of the forest is searched for. Mathematically, the objective function can be defined as

$$
\begin{gathered}
\operatorname{Maximize}\left\{\operatorname{Min}\left\{\frac{\sigma_{t}}{\sigma_{c}}\left(\psi_{r}, \chi_{r}: \psi_{t}, \chi_{t}\right)\right\}_{m}\right\}_{p} \\
\forall m \in 1,2, \ldots, N_{R}
\end{gathered}
$$


TABLE I

THE OPTIMUM POLARIZATION STATES, DERIVED BY GENETIC ALGORITHM USING 20 DIFFERENT SEEDS FOR INITIAL POPULATION SETS OF 200 CHROMOSOMES FOR MAXIMIZATION OF TARGET TO CLUTTER BACKSCATTERING RCS RATIO.

\begin{tabular}{|c|c|c|c|c|c|}
\hline & $\psi_{r}$ & $\chi_{r}$ & $\psi_{t}$ & $\chi_{t}$ & $\operatorname{Max}\left\{\operatorname{Min}\left\{\sigma_{t} / \sigma_{c}\right\}\right\}$ \\
\hline \hline 1st Solution & $25.71^{\circ}$ & $-1.0^{\circ}$ & $36.0^{\circ}$ & $6.0^{\circ}$ & $-6.81 \mathrm{~dB}$ \\
2nd Solution & $-36.0^{\circ}$ & $-10.0^{\circ}$ & $-21.31^{\circ}$ & $2.0^{\circ}$ & $-7.17 \mathrm{~dB}$ \\
\hline
\end{tabular}

TABLE II

MINIMUM TARGET TO CLUTTER BACKSCATTERING RCS RATIO FOR H- AND V- POLARIZATIONS.

\begin{tabular}{|l|l|}
\hline & $\operatorname{Min}\left\{\sigma_{t} / \sigma_{c}\right\}$ \\
\hline \hline$H-$ Polarization & $-18.41 d B$ \\
$V-$ Polarization & $-15.18 d B$ \\
\hline
\end{tabular}

subject to

$$
\psi_{r}, \psi_{t} \in\left[-90^{\circ}, 90^{\circ}\right] \quad \chi_{r}, \chi_{t} \in\left[-45^{\circ}, 45^{\circ}\right]
$$

where $\psi$ and $\chi$ are the orientation and ellipticity angles, respectively. Here $m$ is the realization number and $N_{R}$ is the total number of realizations of the forest. For the same metallic target used in the previous example, with length of $5 \lambda$ along $\hat{y}$ direction and $N_{R}=10$ realizations of forest, optimization of (4) is performed for an incident field propagating along $\theta_{i}=35^{\circ}$, and $\phi_{i}=180^{\circ}$. For the GA procedure an initial population of 200 chromosomes is considered and input vector space is discretized into $2^{26}$ points. Two sets of solution, almost symmetric with respect to $\hat{v}$ direction, are obtained. The optimum values of the polarization angles are shown in table $\mathrm{I}$. The results indicate that using the optimum polarization state, the maximum backscattered RCS ratio of target to clutter of $-6.81 \mathrm{~dB}$ is achieved for a radar having a footprint of about $200 \mathrm{~m}^{2}$. Table II shows the signal-to-clutter ratio when the polarization configuration is simply $\mathrm{V}$ - or $\mathrm{H}$ - polarizations. In comparison, we can achieve about $10 d B$ improvement, using the optimum polarizations.

\section{REFERENCES}

[1] Yi-Cheng Lin and Kamal Sarabandi, A Monte Carlo Coherent Scattering Model For Forest Canopies Using Fractal-Generated Trees, IEEE Trans. Geosci. Remote Sensing, vol. 37, pp. 440451, Jan. 1999.

[2] Il-Suek Koh, and Kamal Sarabandi, Polarimetric Channel Characterization of Foliage for Performance Assessment of GPS Receivers Under Tree Canopies, IEEE Trans. Antennas Propagat., Vol. 50, No. 5, May 2002.

[3] Kamal Sarabandi, and Eric S. Li, Characterization of Optimum Polarization for Multiple Target Discrimination Using Genetic Algorithms, IEEE Trans. Antennas and Propagation. , Vol. 45, No. 12, December 1997.

[4] Mojtaba Dehmollaian, and Kamal Sarabandi, A forward scattering model for foliage camouflaged complex targets, Proceedings on IEEE Geoscience and Remote Sensing, Vol. 1, Sept. 2004.

[5] F. T. Ulaby and M. A. El-Rayes, Microwave dielectric spectrum of vegetation, Part II: Dualdispersion model, IEEE Trans. Geosci. Remote Sensing, vol. GE-25, No. 5, Sep. 1987. 